\title{
Defining and characterizing wood-laden flows in rivers using home videos
}

\author{
Ruiz-Villanueva V. ${ }^{1,}{ }^{*}$, Bürkli, $L^{1}$., Mazzorana B. ${ }^{2,3}$, Mao L. ${ }^{4}$, Ravazzolo D. ${ }^{5,}{ }^{6}$, Iribarren $P .^{2}$, \\ Wohl E. ${ }^{7}$, Nakamura F. ${ }^{8}$, Stoffel M.1, 9 \\ ${ }^{1}$ Institute for Environmental Sciences, University of Geneva, Geneva, Switzerland \\ ${ }^{2}$ Instituto de Ciencias de la Tierra, Faculty of Science, Universidad Austral de Chile, Valdivia, Chile \\ ${ }^{3}$ Universidad Austral de Chile, RINA - Research Unit on Natural and Anthropogenic Risk, Valdivia, \\ Chile \\ ${ }^{4}$ Department of Ecosystems and Environment, Pontificia Universidad Católica de Chile, Santiago, Chile \\ ${ }^{5}$ Hydraulic and Environmental Engineering Department, Pontificia Universidad Católica de Chile, \\ Santiago, Chile \\ ${ }^{6}$ Marine Energy Research and Innovation Center (MERIC), Santiago, Chile \\ ${ }^{7}$ Department of Geosciences, Colorado State University, Fort Collins, CO 80523, USA \\ ${ }^{8}$ Laboratory of Forest Ecosystem Management, Department of Forest Science, Graduate School of \\ Agriculture, Hokkaido University, Kita 9 Nishi 9, Kita-ku, Sapporo, Hokkaido 060-8589, Japan \\ ${ }^{9}$ Department of Earth Sciences, University of Geneva, Geneva, Switzerland
}

\begin{abstract}
Organic material (i.e., trees, branches, wood in general) is commonly neglected among the classical criteria to distinguish flow types, mostly due to the lack of direct observations of flows in which this load is significant. However, in forested basins, large amounts of wood can be transported. Here we define and characterize, for the first time, wood-laden flows and show that these flows may occur repeatedly in a river basin, carrying substantial amount of organic material, with probable impacts on the ecosystem and potential hazards. We propose a novel classification of flows from clear water to hypercongested wood debris flows, according to the composition of sediment, wood and water. Our results will contribute to a better understanding of flow phenomena in forested river basins, which may facilitate the design of better management strategies.
\end{abstract}

\section{Introduction}

Various types of flows involving water and sediments occur in mountain areas, including floods (with or without intensive bedload transport), hyperconcentrated flows and debris flows. However, rivers do not only carry clear water and sediments. In forested basins, large amounts of organic material (i.e., entire trees, logs, branches, root wads, and smaller wood) can be transported as well $[1,2,3]$. The classical criteria used to distinguish between flows may vary, but usually consider inorganic sediment as the only component of the solid fraction and thus neglect the role of organic material $[4,5,6]$. However, the physics of wood transport differs significantly from that of sediment transport because of differences in shape, density and size of the mobile constituents. Therefore, specific characteristics can be expected when flows carry significant amounts of organic material, but these characteristics are not yet well understood. An important limitation in defining organic sediment-laden flows (defined here as wood-laden flows) is the lack of direct observations of flows in which organic material is significant.

\footnotetext{
* Corresponding author: virginia.ruiz@unige.ch
} 
This work aims to shed light on this by collecting data from direct observations of woodladen flows from home videos and by extracting qualitative and quantitative information for a better characterization of flows in forested rivers.

\section{Methodology}

Home videos available in internet were collected by searching online using technical keywords related to floods, flash floods and wood loads in English, German, Spanish, French, and Japanese. More than 45 home videos were retrieved showing floods that occurred in several regions of the world with a notable amount of wood. From all, twenty-eight were selected for further analyses after an initial screening (Figure 1 shows examples).
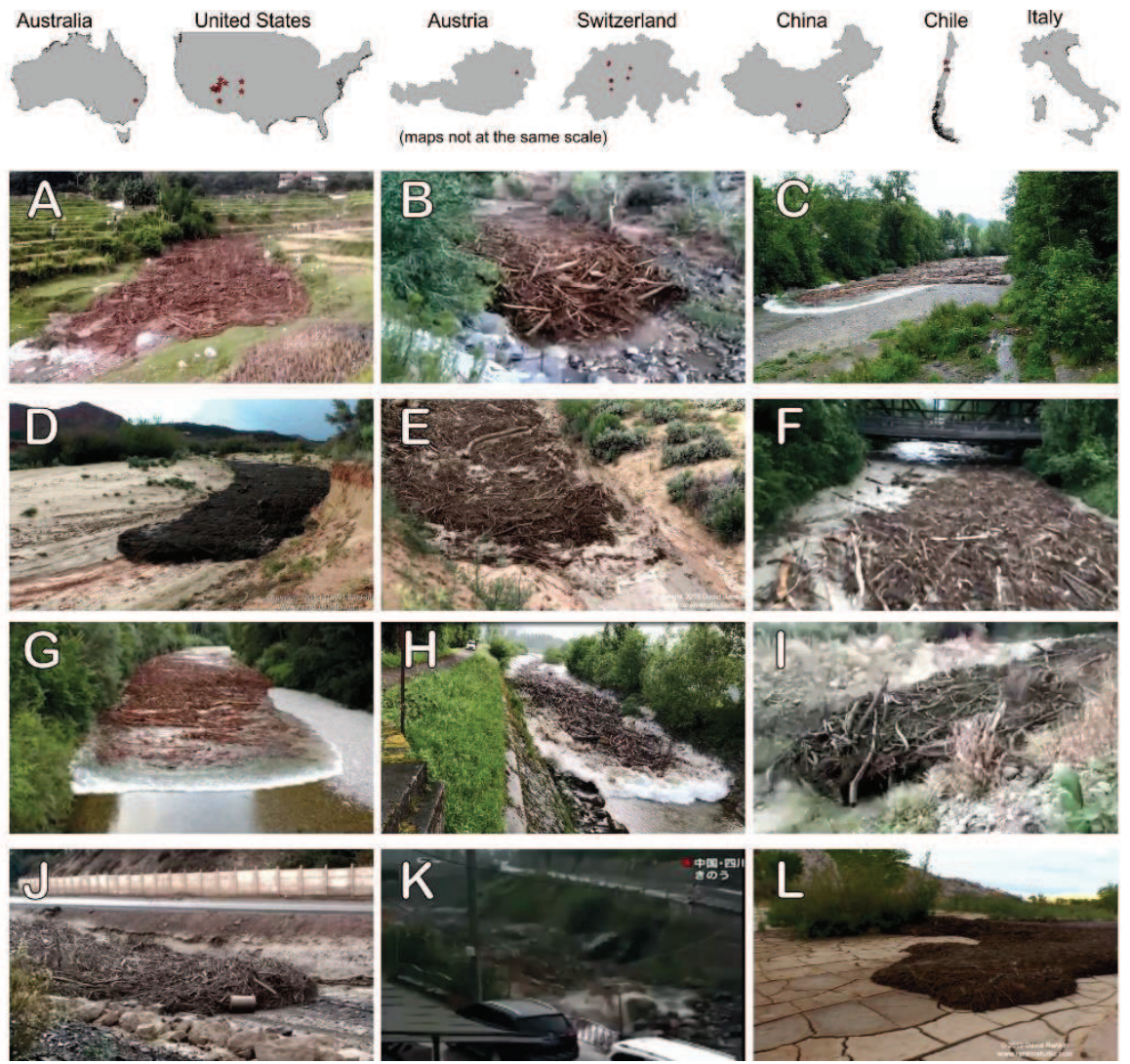

Fig. 1: Images of wood-laden flows extracted from the 28 home movies analyzed in this work. The location of some of the videos is shown in the maps in the lower part of the figure. A Unknown, Unknown; B North Creek, USA; C Zulg River, Switzerland, USA; D Buckskin Wash, USA; E Johnson Canyon, USA; F Zulg, Switzerland; G Emme, Switzerland; H Laui, Switzerland; I Pocuro, Chile; J Quebrada de Marquesa, Chile; K Unknown, China; L Escalante River or tributary, USA.

The location of each video was identified by the metadata or contacting the publisher and thus, watershed and stream characteristics were also collected. For some sites (i.e., videos recorded in Switzerland), ground control points were acquired in the field using a differential GPS. When no field survey was possible, available high-resolution satellite images from Google Earth were used to obtain control points. This process allowed us to extract metrics 
and variables from the frames, such as flow velocity, size of logs, and from the streams and watersheds where videos were recorded (e.g., width and height of the bankfull channel). Watershed characteristics such as drainage area were obtained from Stream Stats (USGS), the dataset from Hydrosheds (USGS) including flow accumulation raster files, and the topographical catchment areas of Swiss waterbodies geodata set from Swisstopo.

Several frames were extracted from the videos and individual frames (1 frame per second) were analysed. Frames were extracted by using Free Video to JPG Converter, Pelscope and Tracker software. In some cases, additional improvements were achieved such as frame stabilization or orthorectification. For these processes, Gimp, Digimizer, CorelDRAW and Fudaa-LSPIV were used.

\section{Preliminary results}

Sites where the wood-laden flows were recorded were classified as Temperate (Cfa, Csa and Cwc), Arid (Bsk and Bwk) and Cold (Dfb, Dfc, Dsc and Dwa) according to [7]. Therefore, wood-laden flows occur within very different climatic zones. Videos were recorded in very diverse sites, from remote and unpopulated areas to urbanized areas and towns, illustrating that these flows may pose significant risk as well.

The obtained results showed that, in general, wood-laden flows were recorded in smallto medium-sized catchments and confined to relatively steep, single thread channels. One important common characteristic is the flashy response, with a very fast (i.e., few minutes) increase in water level, from nearly dry channels to overbank flooding.

The post-processing of these videos provided data to characterize the flows and to redefine wood transport regimes. The classical description of wood transport in rivers considers congested, semi- and uncongested transport [8]. Based on our observations, we added a new regime called hypercongested (Figure 2 ).

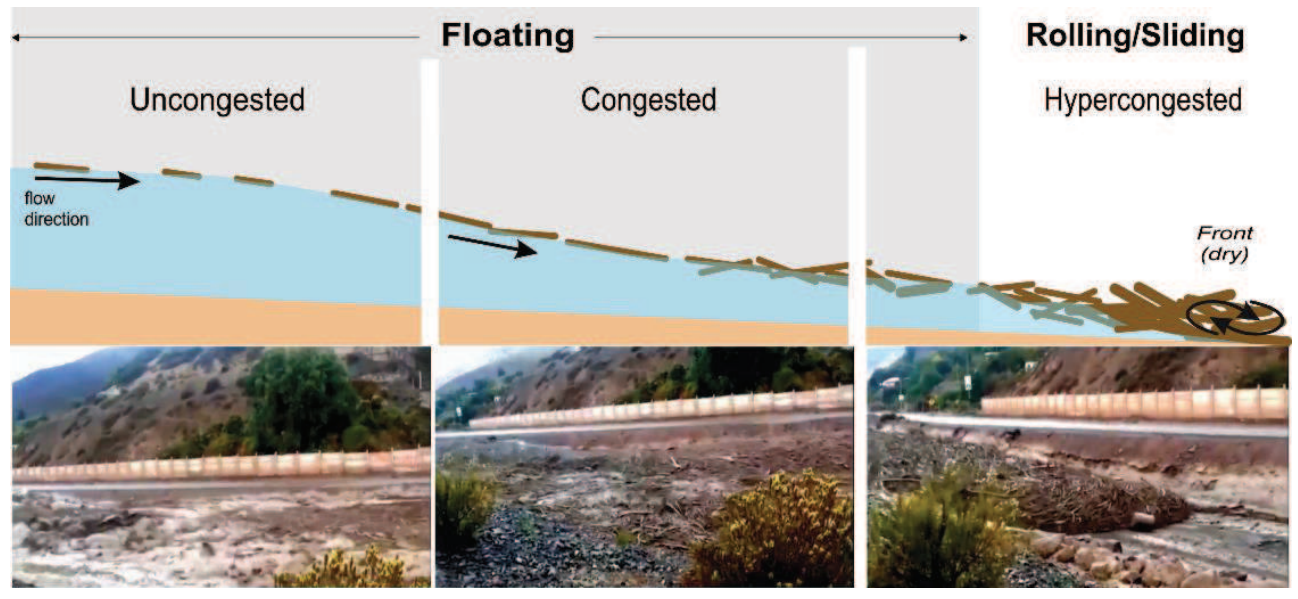

Fig. 2: Wood-laden flow transport regimes. Images from the video recorded in Quebrada de Marquesa (Chile).

We define natural wood transport regimes in terms of three flow regimes: (i) hypercongested regime: a moving log mass occupying the entire wetted area of the crosssection, with full piece-to-piece contacts, and logs rotating or pivoting in the mass. The bulk density of the wood is significantly greater than the water. Wood pieces are collisional and frictional stresses dominate, and the flow is likely moving similar to a rock avalanche or debris flow boulder front; (ii) Congested regime: this is the well-known congested regime 
where floating logs touch each other while moving and where logs occupy a large part of the cross-sectional area. During this regime, the water content is much higher than during hypercongested regime, likely increasing the solid-fluid interactions and the role of viscous forces in the motion of the flow; (iii) Uncongested regime: in this phase logs float and do not interact with each other. They occupy a small part of the cross-sectional area.

These observations allowed us to propose a novel classification based on the properties and characteristics of the flows, represented as a ternary diagram with several boundaries defining the transition from clear water to hypercongested wood debris flows, according to the composition of the mixture (sediment, wood, and water).

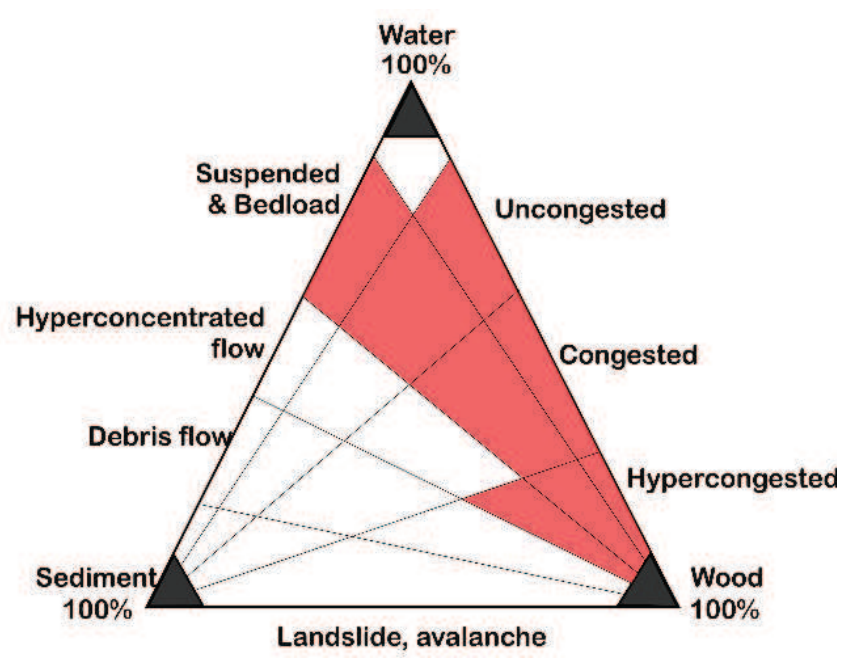

Fig. 3: Ternary diagram of sediment-water-wood with wood-laden flows identified in the videos analyzed in this work colored in red.

The type of ternary diagrams is commonly used to classify sediment-laden based on the proportion of fine and coarse sediment and water. Here we grouped sediment in one single component. The exact values of proportions or percentages of sediment, water and wood may require further analyses.

These flows are natural processes, but the videos alone did not allow inference of the triggering mechanisms. Thus, we hypothesize that wood-laden flows may be naturally generated by one (or a combination) of these four processes:

(i) a large amount of wood within the channel is available for transport before the flashy hydrograph;

(ii) a wood jam breaches, in which a large wood jam collapses and delivers a lot of wood;

(iii) an episodic input of wood from a landslide, debris flow, or a tributary in flood before the arrival of the flood in the main stem;

(iv) the presence of a narrow river section upstream in the main channel, in which the wood might be retained, and suddenly released during the flood.

However, how and where wood comes from during an extreme event remains largely unknown, and wood-laden flows should be monitored more closely. 


\section{Discussion and Conclusions}

This work aimed at describing wood-laden flows, which to our knowledge, have not been described yet as a particular type of flow. Our study illustrates some basic characteristics of these flows but also highlights the need for further research to understand the physics, mechanics and origin of these flows.

River and flood hazard management needs to know whether a river has the capacity to form and propagate wood-laden flows, and thereby cause hazards and impacts on the ecosystem. When this is possible, approaches and measures to analyze and prevent flood hazards should be adapted to the nature of the identified flow. Our results represent a valuable contribution to a better understanding of flow phenomena in forested river basins and will help to design better management strategies.

Acknowledgements: This work was supported by the WoodFlow project funded by the Swiss Federal Office for the Environment (FOEN). Bruno Mazzorana has been supported by the Iniciativa Cientifica Milenio (ICM) through grant NC160025 "Millennium Nucleus CYCLO The Seismic Cycle Along Subduction Zones". We are grateful to all the people who made the videos available, and those who replied our request for permission and additional data.

\section{References}

1. Ruiz-Villanueva, V., Piégay, H., Gurnell, A.M., Marston, R.A., Stoffel, M. Recent advances quantifying the large wood dynamics in river basins: New methods and remaining challenges. Rev. Geophys., 54, 611-652 (2016).

2. Comiti, F., Lucía, A., Rickenmann, D. Large wood recruitment and transport during large floods: a review. Geomorphology, 269, 23-39 (2016).

3. Mazzorana, B., Ruiz-Villanueva, V., Marchi, L., Cavalli, M., Gems, B., Gschnitzer, T., Mao, L., Iroumé, A., Valdebenito, G. Assessing and mitigating large wood-related hazards in mountain streams: recent approaches. J. Flood Risk Manag., (in press), doi:10.1111/jfr3.12316 (2017)

4. Pierson, T.C. and Costa, J.E. A rheologic classification of subaerial sediment-water flows. In: Debris Flows/Avalanches: Processes, Recognition, and Mitigation (J.E. Costa and G.F. Wieczorek, eds). GSA Rev. Engng. Geol.,7, 1-12 (1987).

5. Coussot, P. \& Meunier, M. Recognition, classification and mechanical description of debris flows. Earth-Science Reviews, Vol. 40, No.3-4, pp. 209-227 (1996).

6. Cruden, D.M., Varnes, D.J. Landslide types and processes. In: Turner AK, Schuster RL (eds) Landslides: investigation and mitigation (Special Report). Washington, DC, USA: National Research Council, Transportation and Research Board Special Report 247, pp 36-75 (1996)

7. Peel, M. C., Finlayson, B. L., and McMahon, T. A. Updated world map of the KöppenGeiger climate classification, Hydrol. Earth Syst. Sci., 11, 1633-1644 (2007).

8. Braudrick, C. a., Grant, G.E., Ishikawa, Y., Ikeda, H. Dynamics of wood transport in streams: a flume experiment. Earth Surf. Process. Landforms, 22, 669-683 (1997). 